\title{
Analysis of asphalt wettability based on spreading radius
}

\author{
Peng Tian ${ }^{1,2}$, Lei $\mathrm{Nie}^{1^{*}}$, Gaofeng Zhan ${ }^{1,2}$ \\ ${ }^{1}$ College of Construction Engineering, Jilin University, Changchun 130026, China \\ ${ }^{2}$ Jilin Jianzhu University, Changchun 130118, China
}

Corresponding Author Email: nielei@jlu.edu.cn

https://doi.org/10.18280/ijht.360119

Received: 20 August 2017

Accepted: 19 October 2017

\section{Keywords:}

viscous fluid, wetting, spreading, adhesion ability, interfacial tension, surface energy.

\begin{abstract}
As asphalt is a viscous liquid, its wetting effect on the surface of gravel directly affects the performance of asphalt mixture. Based on viscous fluid mechanics and surface energy theory, the asphalt spreading process on the gravel surface is analyzed. The results show that the spreading ability of asphalt on the solid surface is directly related to the roughness and complexity of the solid surface. At the mixing temperature, the asphalt can spread freely on the gravel surface. Its adhesion ability on the gravel surface can be evaluated by observing its spreading radius. After the asphalt droplets land on the gravel surface, and being heated for 3 minutes, the spreading is completed and the free energy balance of solid, liquid and gas surfaces is achieved. The limestone gravel has the roughest surface, the most complex pore features on surface, and the smallest spreading radius. The surface roughness of basalt gravel is basically the same as that of andesite surface.
\end{abstract}

\section{INTRODUCTION}

Asphalt is an indispensable building material for road construction. Asphalt, a kind of high viscosity organic liquid, is a black-brown complex mixture composed of hydrocarbons with different molecular weights and their non-metallic derivatives. Asphalt becomes solid or semi-solid at low temperature while liquid at high temperature [1]. During the asphalt mixture production process, it is necessary to keep the temperature of asphalt and aggregate [2] within the reasonable mixing temperature range of $135-170^{\circ} \mathrm{C}$ in order to maintain its liquid state. At this time, the asphalt is in a high viscosity liquid state.

In the road engineering field, the adhesion ability of asphalt on the gravel surface directly affects the performance of road with asphalt mixture [3], which can be measured and evaluated by water boiling test, water immersion test and photoelectric colorimetric test. However, the traditional experimental methods are often influenced by people's subjective consciousness [4]. There is viscosity in asphalt flow, which is the macroscopic expression of momentum transfer caused by molecular thermal motion and intermolecular force. With the burgeoning process in surface energy theory, more and more experts analyze the surface interface effect of liquid on solid surface [5-7]. The adhesion of asphalt on the gravel surface can be analyzed by wettability experiment, with major wettability measurement methods including contact angle method [8], imbibition and displacement method, microscope observation and so on. With the development of experimental imaging technologies such as dynamic imaging, contact angle detectors [9], and capillary rise detection, and the development of microscopic image analysis and surface energy theory [1112], the research on the adhesion property of asphalt on the surface of gravel has been increasingly studied in-depth.

In this paper, 90 \# asphalt is used as test liquid to test its spreading on three kinds of solid surfaces with different levels of roughness. Based on the theory of viscous fluid mechanics and surface energy, the spreading process of asphalt on the gravel surface is analyzed by observing the spreading radius with microscope.

\section{WETTABILITY}

\subsection{Wettability theory}

Wettability refers to the ability or propensity of a liquid to spread on a solid surface [13]. The wetting of a liquid on a solid surface includes three processes of damping, soaking, and spreading [14]. Let $\gamma_{l g}$ be the surface gas free energy, and $\gamma_{s l}$ be the solid-liquid surface free energy. Damping is the process by which a liquid comes into contact with a solid surface. The variation of free energy $\Delta G$ under constant temperature and constant pressure is shown in Formula (1). Soaking refers to the process of immersing a solid into a liquid, during which the solid-air interface is replaced by a solidliquid interface, and the liquid surface does not change. Therefore, the variation of the free energy $\Delta G$ in this process is as shown in Formula (2). The essence of the spreading process is the replacement of the gas-liquid interface with the solid-liquid interface, and the expansion of the gas-liquid interface. The system free energy reduction value $\Delta G$ can be expressed by Formula (3).

$$
\begin{aligned}
& \Delta G=\gamma_{s g}+\gamma_{l g}-\gamma_{s l} \\
& \Delta G=\gamma_{s g}-\gamma_{s l} \\
& \Delta G=\gamma_{l g}+\gamma_{s l}-\gamma_{s g}
\end{aligned}
$$

When $\Delta G<0$, each process will proceed spontaneously. 
For the wetting process that forms a certain contact angle, there is the following relationship between the above threeplane (table) surface tension and the contact angle $\theta$.

$\gamma_{s g}-\gamma_{s l}=\gamma_{l g} \cos \theta$

The formula of spreading the real number $\mathrm{S}$ can be obtained by substituting Formula (4) into Formula (3), take Formula (5) as an example.

$S=\gamma_{l g}(\cos \theta-1)$

Formulas (4) and (5) are basic equations describing the wetting degree. When $\theta=0^{\circ}, \mathrm{S}=0$, and it is a complete wetting state, then the liquid can spread automatically on the solid surface. When $\theta=180^{\circ}$, it is a complete non-wetting state, the droplets form an ideal sphere on the solid surface; When $0^{\circ}<\theta<180^{\circ}$, it is an incomplete wetting state, the smaller the $\theta$, the easier for the liquid to wet the solid surface. It is generally considered that when $\theta>90^{\circ}$, the liquid does not wet the solid surface, while when $\theta<90^{\circ}$, the liquid wet the solid surface. [15]

\subsection{Spreading radius of droplets}

The adhesion work, adhesion tension and spreading coefficient are usually calculated by measuring the surface tension and contact angle of the liquid [16-17]. Upon the need of measuring technology, the traditional methods for measuring contact angle must grind the solid (aggregate) surface flat and fine. The aggregate plane obtained by grinding has lost many of the features of the original aggregate surface in engineering practice. In order to obtain the most realistic spreading effect of asphalt on the aggregate surface, only the relatively flat original surface of gravel is selected as the experimental surface, for which no grinding treatment is done, except for cleaning surface impurities.

The gravel surface is rough and uneven, with a rich capillary system, which has a complicated influence on the wetting process of asphalt. For the capillary system with uniform pore diameter, when the contact angle $\theta<90^{\circ}$, the wetting effect of liquid on the inner wall of the pores is reflected in the automatic filling of asphalt in the capillary structure. That is, the liquid can penetrate into the pores driven by the pressure difference of the curved surface [18]. The pore of gravel aggregate surface has various diameters, different angles, diverse sizes and uneven distribution, which makes the wetting process of asphalt more complicated. It is necessary to consider not only the effect of surface energy but also the influence of the distribution density, aperture size, inner wall shape and aperture direction of the capillary system. It is necessary not only to consider the role of surface energy, but also to consider the distribution density of the pore system, the pore size, the shape of the inner wall and the direction of the pore size. In summary, the aggregate surface is extremely undesirable, and it is difficult to measure the contact angle. Therefore, an attempt is made to evaluate the wetting level of asphalt on the aggregate surface by methods other than measuring contact angle.

In a view to avoiding the errors in the measurement of contact angle, Zhao Zhenguo [19] proposes to calculate the potential energy variation and the surface free energy variation of the liquid cake by measuring its height variation. However, this method requires the liquid to be continuously filled for a period of time, and the height of the liquid cake can only be determined when the height of the liquid cake is maintained constant. This method has also been tried in this paper. However, the asphalt is rapidly cooled and condensed during the dropping process after leaving the environment of mixing temperature $\left(150{ }^{\circ} \mathrm{C}\right)$ of the thermostat as the asphalt liquid is very sensitive to the temperature [20]. Thus it is impossible to achieve the continuous filling of the asphalt droplets, and it is difficult to reach the saturated liquid cake state of the asphalt droplets required for liquid cake height method. It indicates that the method does not apply to asphalt that is semi-solid at normal temperature.

Based on these reasons, this paper proposes to determine the spreading coefficient of asphalt on the aggregate surface by measuring the spreading radius, and to analyze the wetting effect of asphalt on various aggregate surfaces.

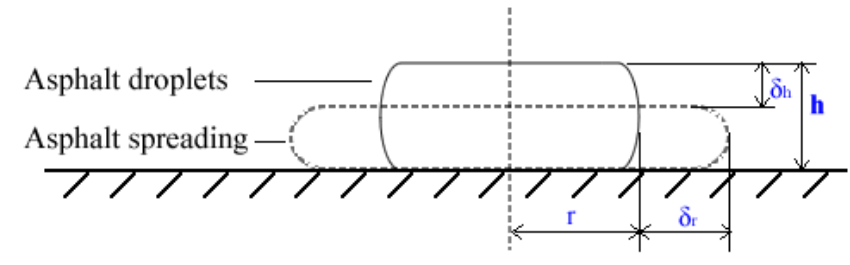

Figure 1. Profile of liquid cake spreading

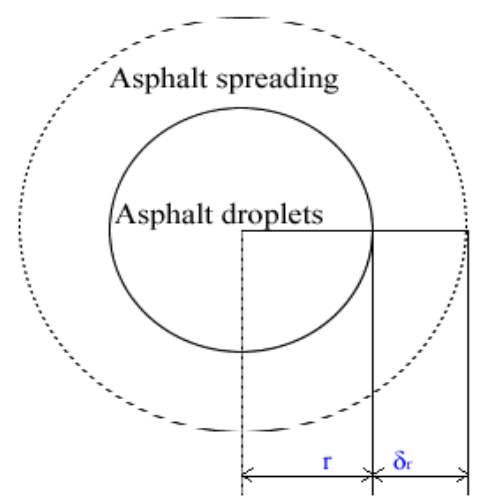

Figure 2. Plane of liquid cake spreading

Figure 1 shows the profile of liquid cake spreading. Let the cake volume be $V$, limit height $h$, and radius $r$. If the equilibrium liquid cake expands by a minimal amount $\delta_{r}$, the height will decrease by $\delta_{h}$, and the surface free energy during this process changes to $2 \pi r \delta_{r}\left(\gamma_{l g}+\gamma_{s l}-\gamma_{s g}\right)$. Since the liquid cake height decreases by $\delta \delta_{h}$, the liquid cake potential decreases by $m g \delta_{h} / 2$ or $\rho g V \delta_{h} / 2$. Where, $m$ is the mass of the liquid cake, $\rho$ is its density, and $g$ is the gravitational acceleration.

The above process is a very slight change under the equilibrium conditions, so the surface free energy change is equal to the potential energy change, i.e.

$2 \pi r \delta_{r}\left(\gamma_{l g}+\gamma_{s l}-\gamma_{s g}\right)=\frac{1}{2} \rho g V \delta_{h}$

As shown in Figure 2, there is no change in the total volume of the liquid cake during this process, and the asphalt liquid cake is circularly spread out in a circle, as shown in Formula (7). 
$\pi\left(\left(r+\delta_{r}\right)^{2}-r^{2}\right)\left(h-\delta_{h}\right)=\pi r^{2} \delta_{h}$

When Formula (7) is substituted into Formula (6), it can be obtained.

$S=-\frac{1}{2} \rho g h^{2}$

Thus, the relation between $\theta$ and $h$ can be obtained as in Formula (9)

$\cos \theta=1-\frac{\rho g h^{2}}{2 \gamma_{l g}}$

It can be seen that the contact angle of the droplets on the surface of the aggregate can be deduced by measuring the spreading radius of the asphalt droplets. That is, the spreading coefficient and adhesion ability of asphalt on the surface of aggregate can be determined by the spreading radius of asphalt droplets on the surface of aggregate.

\section{EXPERIMENT OF SPREADING}

\subsection{Basic properties of asphalt}

The asphalt used in the experiment is 90 \# asphalt produced by Liaoning Panjin Northern Asphalt Co., Ltd. Its performance indicators are as shown in Table 1.

Table 1. List of basic properties of asphalt

\begin{tabular}{cc}
\hline Items & 90\#Asphalt \\
\hline Needle penetration $\left(25^{\circ} \mathrm{C}, 100 \mathrm{~g}, 5 \mathrm{~s}, 0.1 \mathrm{~mm}\right)$ & 86 \\
Ductility $5^{\circ} \mathrm{C}$ & 150 \\
Softening point & 45 \\
Solubility $(\%)$ & 99.97 \\
Flashing point $\left(\right.$ hatch, $\left.{ }^{\circ} \mathrm{C}\right)$ & 302 \\
Density $\left(25^{\circ} \mathrm{C}, \mathrm{kg} / \mathrm{m} 3\right)$ & 1000.3 \\
Wax conten $\mathrm{t}($ Distillation, $\%)$ & 1.89 \\
\hline
\end{tabular}

\subsection{Spreading experiment}

Asphalt is very sensitive to changes in temperature [21-22]. The change of the state of the bitumen droplet can be observed on the microcosmic image. After the aggregate and asphalt are heated to the mixing temperature $\left(150^{\circ} \mathrm{C}\right)$ in the thermostat, the asphalt is in the liquid state. Even if the distance[23] of drop is very close, during the process of droplets falling on the surface of the stone, the temperature of the asphalt droplets rapidly decreases and the droplets tend to condense, as shown in Figure 3. The edges of the asphalt droplets are neat and there is no spreading phenomenon, as shown in Figure 4.

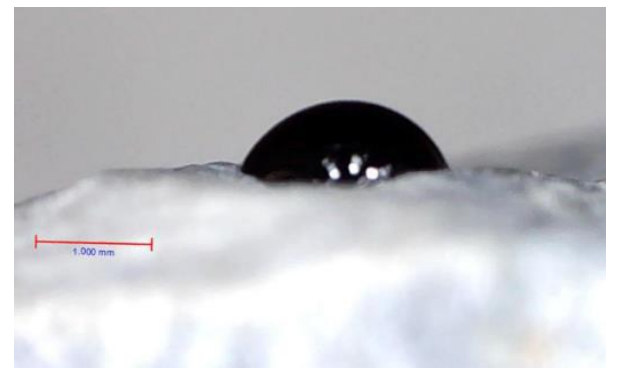

Figure 3. Properties of asphalt droplets on aggregate surface

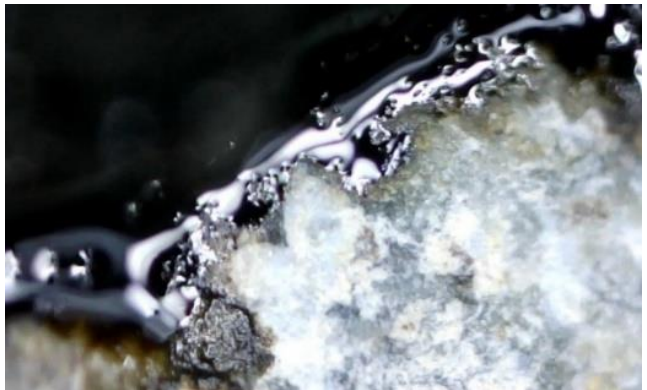

Figure 4. Edges of asphalt droplets on aggregate surface

Thus, after the asphalt drops off, the specimen is again placed in a thermostat for 5 minutes, with the temperature restored to the mixing temperature. During this heating process, the surface of the aggregate with the asphalt droplets is kept horizontal.

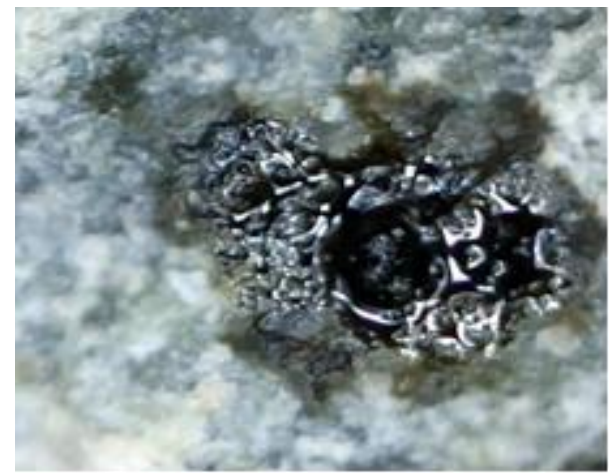

Figure 5. Spreading of asphalt droplets after being heated

As can be seen from Figure 5, as the temperature rises and the heating time increases, the asphalt droplets can spread freely, which means, there appears the spreading phenomenon of asphalt on the surface of aggregate. Therefore, the complexity of the aggregate surface can be assessed by measuring the spreading level of asphalt on the aggregate surface. For example, as shown in Figure 1 and Figure 2, the change in asphalt liquid cake height is $\delta_{h}$ and the change in liquid cake diameter is $\delta_{r}$ after the asphalt droplets spread. In the present study, the potential energy change of asphalt cake is calculated by measuring the diameter change of the cake.

\subsection{Measurement of the spreading radius}

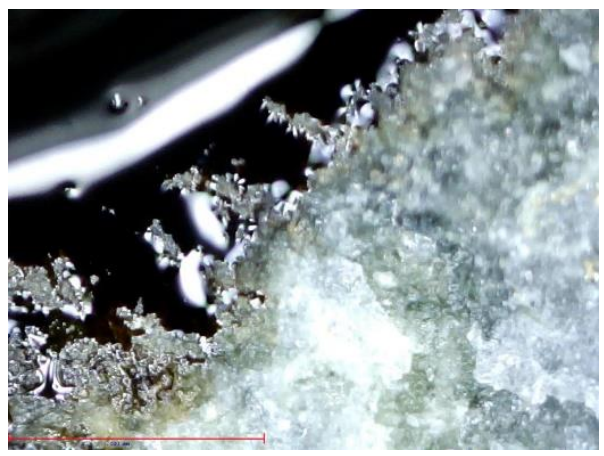

Figure 6. Asphalt dripping on basalt aggregate surface

The aggregate specimen shall be placed on the adjustable platform and the observation shall be maintained at a plane level. As asphalt is heated to the mixing temperature $\left(150^{\circ} \mathrm{C}\right)$, 
a large amount of asphalt drops onto the aggregate surface. After the asphalt drops onto the aggregate surface, the asphalt rapidly condenses into droplets due to the rapid decrease in temperature, with neat edges and no obvious spreading, as shown in Figure 6.

After the specimen is heated in a thermostat for 1 minute, spreading appears, as shown in Figure 7. It shows that asphalt has spreading ability on the surface of aggregate, and the pore, texture and capillary system on the gravel surface play a significant role in the spreading process.

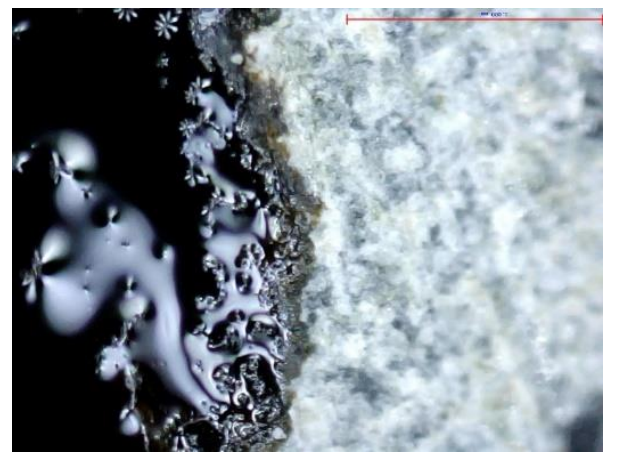

Figure 7. Asphalt droplets on the surface of basalt aggregate after being heated for 1 minute

After the asphalt droplets being heated for 3 minutes, their spreading becomes more obvious, as shown in Figure 8. Asphalt droplets become noticeably thinner, mottled immersions appear, and the spreading radius continues to expand.

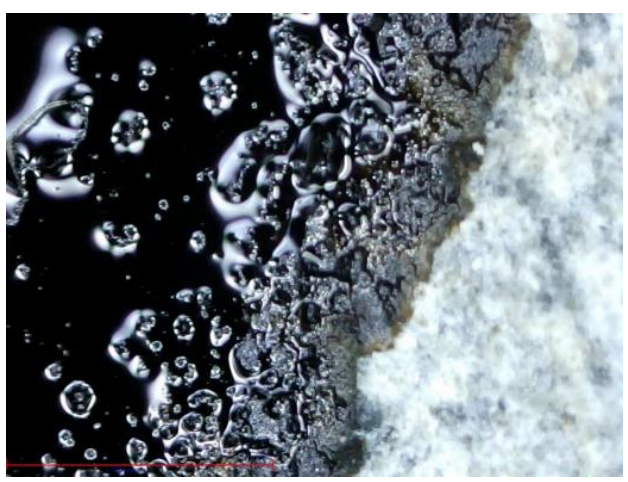

Figure 8. Asphalt droplets on the surface of basalt aggregate after being heated for 3 minutes

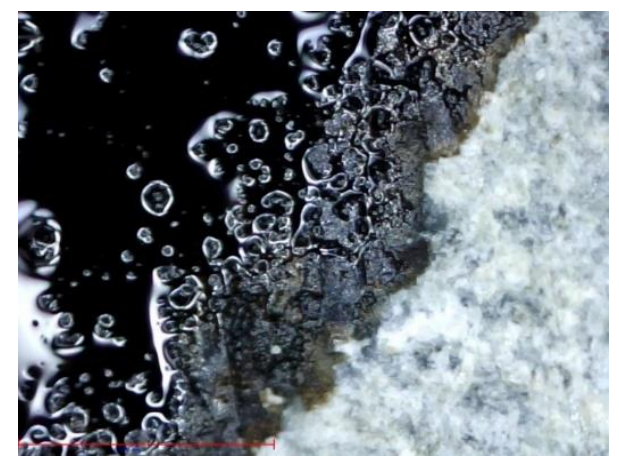

Figure 9. Asphalt droplets on the surface of basalt aggregate after being heated for 5 minutes
The heating is continued for 5 minutes, then the spreading degree of asphalt is similar to that of asphalt after being heated for 3 minutes, and its spreading radius is no longer increased, as shown in Figure 9. It indicates that after being heated for 3 minutes, the spreading of asphalt on the surface of aggregate is completed, and the surface free energy on liquid, solid and gas interface is balanced.

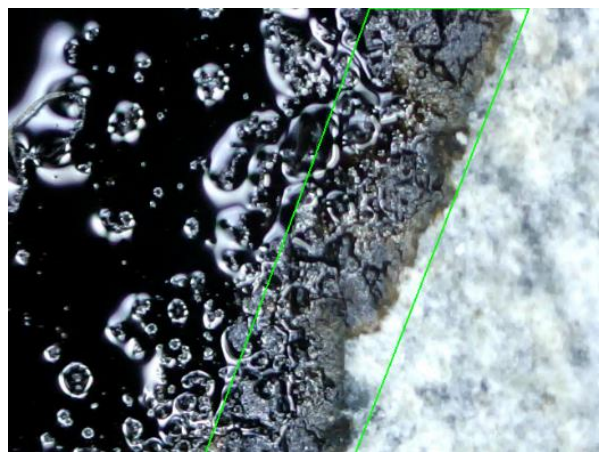

Figure 10. Selection of observation area for basalt asphalt spreading

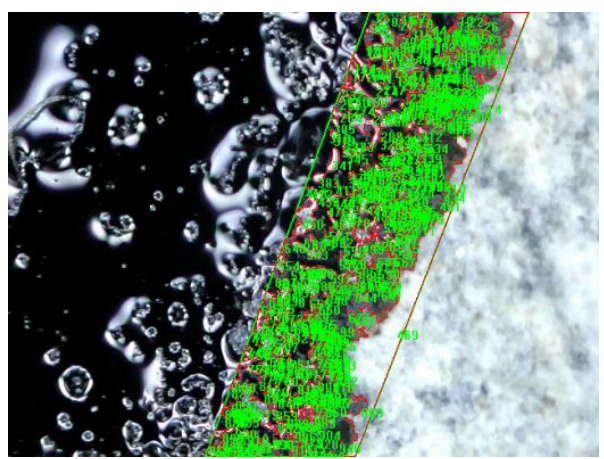

Figure 11. Color counting points in spreading area

According to the spreading area in Figure 10 and the width of the spreading area in Figure 11, the average spreading radius of the observation area can be calculated by the Formula (10).

$\delta_{r}=\frac{s}{l}$

where, $S$ is the spreading area of the measurement area, $l$ is the spreading width of the measurement area, and $\delta_{r}$ is the average spreading radius.

\section{COMPARATIVE ANALYSIS}

Table 2. Spreading radius of 90 \# asphalt on the gravel surface

\begin{tabular}{lcccc}
\hline NO. & Type & $\begin{array}{c}\text { Spreadin } \\
\text { g area } \boldsymbol{S} \\
(\mathbf{m m 2})\end{array}$ & $\begin{array}{c}\text { Spreadin } \\
\text { g width } \boldsymbol{l} \\
(\mathbf{m m})\end{array}$ & $\begin{array}{c}\text { average } \\
\text { spreading } \\
\text { radius } \boldsymbol{\delta}_{\boldsymbol{r}} \\
(\mathbf{m m})\end{array}$ \\
\hline X11 & Basalt & 0.103 & 0.500 & 0.206 \\
X12 & Basalt & 0.097 & 0.442 & 0.219 \\
X13 & Basalt & 0.102 & 0.457 & 0.223 \\
X21 & Basalt & 0.161 & 0.678 & 0.237 \\
X22 & Basalt & 0.146 & 0.647 & 0.225 \\
X23 & Basalt & 0.236 & 0.884 & 0.267 \\
X31 & Basalt & 0.248 & 0.879 & 0.282 \\
\hline
\end{tabular}




\begin{tabular}{lclll}
\hline X32 & Basalt & 0.266 & 0.957 & 0.278 \\
X33 & Basalt & 0.187 & 0.656 & 0.286 \\
A11 & Andesite & 0.182 & 0.704 & 0.259 \\
A12 & Andesite & 0.175 & 0.645 & 0.272 \\
A13 & Andesite & 0.202 & 0.770 & 0.263 \\
A21 & Andesite & 0.365 & 1.121 & 0.326 \\
A22 & Andesite & 0.263 & 0.759 & 0.347 \\
A23 & Andesite & 0.300 & 0.897 & 0.335 \\
A31 & Andesite & 0.194 & 0.691 & 0.281 \\
A32 & Andesite & 0.344 & 0.897 & 0.383 \\
A33 & Andesite & 0.407 & 1.167 & 0.349 \\
S11 & Limestone & 0.062 & 0.362 & 0.171 \\
S12 & Limestone & 0.067 & 0.423 & 0.158 \\
S13 & Limestone & 0.029 & 0.249 & 0.115 \\
S21 & Limestone & 0.044 & 0.321 & 0.137 \\
S22 & Limestone & 0.024 & 0.242 & 0.101 \\
S23 & Limestone & 0.058 & 0.349 & 0.165 \\
S31 & Limestone & 0.032 & 0.292 & 0.108 \\
S32 & Limestone & 0.028 & 0.252 & 0.112 \\
S33 & Limestone & 0.025 & 0.247 & 0.103 \\
\hline
\end{tabular}

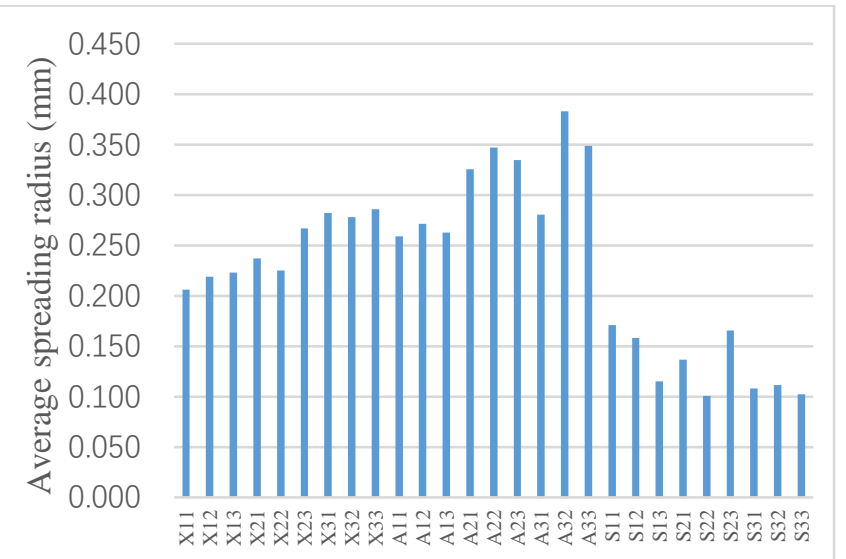

Specimen

Figure 12. Histogram of spreading radius of 90 \# asphalt on gravel surface

As can be seen from Figure 12, the spreading radius of basalt and andesite is larger, and that of limestone is smaller. The surface texture of limestone is rough, with rich pores, which makes it unfavorable for asphalt spreading. This shows that the spreading radius of asphalt on the surface of gravel is inversely proportional to its roughness.

\section{CONCLUSIONS}

At the mixing temperature, the asphalt can spread freely on the gravel surface. After the asphalt droplets land on the surface of the rubble, they are being heated for 3 minutes. Then the spreading is completed and the free energy balance between the solid, liquid, and gas surfaces is achieved. Based on the spreading radius, the spreading degree of asphalt on the gravel surface can be evaluated. The smoother the gravel surface is, the larger its spreading radius will be, while the rougher its surface is, the smaller its spreading radius will be. The limestone gravel has the roughest surface, the most complex surface pore features, and the smallest spreading radius. The surface roughness of basalt gravel is basically the same as that of andesite, and the value of spreading radius is close to that of andesite. In summary, it is feasible to determine the adhesion of asphalt on surfaces with different roughness at any time through spreading radius. Although there are still errors in the observation process, the measurement method can largely approximate the spreading process of the viscous fluid on the rough surface in the actual environment. It is a significant attempt to analyze the flow and wetting properties of viscous fluid on solid surface by spreading radius experiment.

\section{ACKNOWLEDGMENT}

This study was supported by the 13th Five-Year science of the Education Department in Jilin Province, Jilin Education Science Planning Project (GH150340) and technology research project planning 2017 in Jilin provincial industrial innovation special fund project (2017C030-3).

\section{REFERENCES}

[1] Zhou XD. (2013). Temperature sensitivity of rubber modified asphalt research. Science Technology \& Engineering 13(30): 9114-9117. https://doi.org/ 10.3969/j.issn.1671-1815.2013.30.049

[2] Abed A, Thom N, Grenfell J. (2017). Evaluation of mixing temperature impact on warm mix asphalt performance. International Conference on the Bearing Capacity of Roads, Railways and Airfields 251-258. https://doi.org/ 10.1201/9781315100333-37

[3] Tian P, Shukla A, Nie L, Zhan GF, Liu S. (2018). Characteristics relation model of asphalt pavement performance based on factor analysis. International Journal of Pavement Research \& Technology 11(1): 112. https://doi.org/ 10.1016/j.ijprt.2017.07.007

[4] Song D. (2017). Relational decision-making processes. NeuroQuantology 15(3): 120-124. https://doi.org/ 10.14704/nq.2017.15.3.1053

[5] Bhasin A, Little DN. (2007). Characterization of aggregate surface energy using the universal sorption device. Journal of Materials in Civil Engineering 19(8): 634-641. https://doi.org/ 10.1061/(ASCE)0899-1561

[6] Azarhoosh AR, Nejad FM, Khodaii A. (2016). Using the surface free energy method to evaluate the effects of nanomaterial on the fatigue life of hot mix asphalt. Journal of Materials in Civil Engineering 28(10): 04016098. https://doi.org/ 10.1061/(ASCE)MT.19435533.0001614

[7] Mirhosseini SAF, Khabiri MM, Kavussi A, Kamali MHJ. (2016). Applying surface free energy method for evaluation of moisture damage in asphalt mixtures containing date seed ash. Construction \& Building Materials 125: 408-416. https://doi.org/ 10.1016/j.conbuildmat.2016.08.056

[8] Moraes R, Velasquez R, Bahia H. (2017). Using bond strength and surface energy to estimate moisture resistance of asphalt-aggregate systems. Construction \& Building Materials 130: 156-170. https://doi.org/ 10.1016/j.conbuildmat.2016.10.043

[9] Habal A, Singh D. (2017). Moisture damage resistance of gtr-modified asphalt binders containing wma additives using the surface free energy approach. Journal of Performance of Constructed Facilities 31(3). https://doi.org/ 10.1061/(ASCE)CF.1943-5509.0000995 
[10] Mitchell MR, Link RE, Sinkonde D, Hao PW, Xiao QY. (2007). Evaluate the simple surface energy of aggregates using the capillary rise method. Journal of Testing \& Evaluation 35(6): 618-623. https://doi.org/ 10.1520/JTE100973

[11] Mirhosseini AF, Kavussi A, Kamali MHJ, Khabiri MM, Hassani A. (2017). Evaluating fatigue behavior of asphalt binders and mixes containing date seed ash. Journal of Civil Engineering \& Management, 23(8): 1164-1175. $10.3846 / 13923730.2017 .1396560$ https://doi.org/

[12] Kakar MR, Hamzah MO, Akhtar MN, Woodward D. (2016). Surface free energy and moisture susceptibility evaluation of asphalt binders modified with surfactantbased chemical additive. Journal of Cleaner Production 112(4): 2342-2353. https://doi.org/ 10.1016/j.jclepro.2015.10.101

[13] Fanchi JR. (2010). Integrated reservoir asset management. Integrated Reservoir Asset Management 80(5): 355-361. https://doi.org/ 10.1016/C2009-062240-6

[14] Yonemoto Y, Kunugi T. (2014). Wettability model for various-sized droplets on solid surfaces. Physics of Fluids 26(8): 757-772. https://doi.org/ 10.1364/AO.57.002080

[15] Yonemoto Y, Kunugi T. (2014). Wettability model for various-sized droplets on solid surfaces. Physics of Fluids 26(8): 757-772. https://doi.org/ $10.1063 / 1.4893935$

[16] Job G, Rüffler R. (2016). Interfacial phenomena. physical chemistry from a different angle. Springer
International Publishing 87(2): 495-508. https://doi.org/ 10.1007/978-3-319-15666-8_15.

[17] Tsekov R, Borissov D, Karakashev SI. (2013). Wetting dynamics on lyophilic solid surfaces patterned by lyophobic islands. Colloids \& Surfaces 423: 77-80. https://doi.org/ 10.1016/j.colsurfa.2013.02.007.

[18] Schirmer W. (1999) Physical chemistry of surfaces. Zeitschrift Für Physikalische Chemie 210(Part_1):134135. https://doi.org/ 10.1524/zpch.1999.210.Part_1.134

[19] Zhao ZG. (2001). Study of wetting on solid surfaces by measuring height of large sessile drop. Journal of Shangqiu Teachers College 2001(02): 97-99. https://doi.org/ 10.3969/j.issn.1672-3600.2001.02.023.

[20] Alam MS, Islam T, Uddin MJ. (2016). Mathematical modelling for heat transfer of a micropolar fluid along a permeable stretching/shrinking wedge with heat generation/absorption. Mathematical Modelling of Engineering Problems 3(1): 1-9. https://doi.org/ 10.18280/mmep.030101

[21] Kwon H, Lee AS, Jin HL, Park NK, Kim GD, Cho B, et al. (2017). Characterization of liquid state sulfur polymer/epoxy blend as asphalt pavement materials. Journal of Industrial \& Engineering Chemistry 53(25): 386-391. https://doi.org/ 10.1016/j.jiec.2017.05.010

[22] Wang JG, Wang XR, Ren GL, Xiang K. (2017). Effect of ambient condition on $\mathrm{n}$-heptane droplet evaporation, Mathematical Modelling of Engineering Problems 4(1): 13-17. https://doi.org/ 10.18280/mmep.040103

[23] Kocaslan G. (2017). The role of distance in the gravity model: from the view of newton, international economics and quantum mechanics, NeuroQuantology 15(2): 208214. https://doi.org/ 10.14704/nq.2017.15.2.1023 